\title{
Effect of insulin in the induction and regression of experimental cholesterol atherosclerosis in the rabbit
}

\author{
G. MARQUiÉ \\ Département de Physiologie animale et de la Nutrition, Institut de Biologie, \\ Université des Sciences et de la Technologie, Alger, Algérie
}

\begin{abstract}
Summary
A study was undertaken to determine the effects of long-term insulin therapy on the development and regression of lipid perturbations and experimental cholesterol atherosclerosis in rabbits:

(1) Insulin administration for 15 days significantly reduced plasma lipid levels and free fatty acids in rabbits fed a high-cholesterol diet; it also inhibited the effects of a single dose of cholesterol. Paradoxically, continued insulin treatment led to the reinforcement of lipaemia through the stimulation of mobilization. Insulin administration during the development of atherosclerosis significantly aggravated the fatty infiltration of the aortic tissue and the lesions of the vessels, and also increased the frequency of coronary lesions.
\end{abstract}

(2) In rabbits fed a cholesterol enriched diet during two months and then a normal diet, insulin treatment accelerated the rate of reduction of hypercholesterolaemia, but aggravated the lipid infiltration of the artery walls, and also prevented regression of coronary atherosclerosis.

\section{Introduction}

Atherosclerotic vascular complications become the major causes of morbidity and mortality in diabetic persons, doubtless due in part to their increased life span with insulin therapy. Diabetics show an increased susceptibility to atherosclerotic disease. The causative and pathogenic factors in diabetes remain obscure. There is considerable evidence implicating alterations in lipid metabolism in the pathogenesis of diabetic atherosclerosis. Undoubtedly these data demonstrate an association between lipid disorders and atherogenesis in diabetes. The precise relationship of this lipid pathophysiology to diabetic atherogenesis is not known.

Abnormalities in insulin secretion have also been described from patients with ischaemic heart disease (Christiansen et al., 1968; Leetma et al., 1968; Tzagournis et al., 1968). These abnormalities consist in raised insulin responses to oral carbohydrate with or without high fasting plasma insulin levels (Peter's and Hales, 1965). It has been suggested by Stout and Wallance-Owen (1969) that hyperinsulinism may bed the common factor linking atherosclerosis with diaios betes, obesity and hyperlipidaemia. An acute in vitr 8 effect of insulin on the lipid metabolism of the arterial wall has been described (Mahler, 1966; Stout $\overrightarrow{t_{1}}$ 1968, 1969, 1971). Under these circumstances, i seemed reasonable to postulate that insulin mat have a causal role in atherogenesis.

In view of this problem, experiments were under $=$ taken to explore the effects of exogenous insulinow aorta and coronary atherogenesis in cholesterol-fed rabbits and in rabbits after cessation of the chotes terol diet.

\section{Materials and methods}

The rabbits were male of the Fawn Bourgogne strain and New Zealand White strain and weighef approximately $2.5 \mathrm{~kg}$ at the start of the experiment Upon entering the animal house, the rabbits were placed in individual cages and allowed not less that 7 days for acclimatization. Their food consisted of commercial rabbit chow (150 mg daily) and water a $a$. libitum. At least one control determination of thei' serum lipid and blood sugar values was usually made.

The work was carried out on 186 rabbits divide $\Phi$ in the following groups (number of animals shown in parentheses): I-normal diet (18); II-cholesteros $300 \mathrm{mg} / \mathrm{kg}$ body weight (57); III-insulin + choles 7 terol (57); IV-regression after cessation of the. cholesterol-containing diet (29); V-insulin afterv cessation of the diet (25).

Cholesterol was given as follows: cholesterol wa: added to the food as a $10 \% \mathrm{~W} / \mathrm{V}$ solution in ethyd ether. It was rapidly and uniformly mixed with the food pellets which were then allowed to dry untit the odour of ether had gone. The cholesterol containing food was given to each rabbit every morning at the rate of $300 \mathrm{mg} / \mathrm{kg} / \mathrm{day}$. Insulin treat ment began on the day before cholesterol feeding was initiated. Protamine zinc insulin injection 1. $\frac{\text { T }}{0}$ 
u./ $\mathrm{kg}$ was given subcutaneously daily in the morning only. Controls were injected an equal volume of saline. Ingestion of the diet was carefully controlled for each animal.

The duration of cholesterol feeding, with or without insulin treatment, varied from 1 to 90 days. Groups IV and V were kept on a normal diet for a further 120 days, with or without insulin, and sacrificed at different intervals. Blood samples were taken after $1,5,10,15,30,60,90,120$ days of treatment to determine the various lipid constituents of the plasma. As each rabbit was sacrificed, samples were taken of the thoracic aorta, with its adventitious tunica removed, and of the liver for biochemical analysis. Extraction of the plasma or tissue lipids was carried out in accordance with the method of Folch, Lees and Stanley (1957). The quantity of total fatty acids was determined by a micro-method developed from the works of Sperry and Brand (1955), Folch et al. (1957) and Albrink (1959), and that of total cholesterol by the colorimetric method of Lieberman-Burchard described by Stadtman (1957). Lipid phosphorus was determined following the method of Chen, Toribara and Warner (1956), free fatty acids were measured by the method of Laurell and Tibbling (1966) and blood sugar by the method of Somogyi (1952).

The aorta was opened and the degree of atherosclerosis recorded by means of schematic drawing and graded on an arbitrary scale from 0 to 4 (Miller and Kothati, 1969). Samples were taken of the thoracic aorta, the pulmonary artery and the myocardium for histopathological examination. The tissues were stained with Masson's trichrome and Verhoeff-van Gieson phosphotungstic acid haematoxylin in order to confirm the degree of the elastic fibres. The presence and extent of the arterial lesions were evaluated against a normal atherosclerosis scale ranging from 0 to 4 (Anitschkow, 1933).

The data were examined using Student's $t$-test: the deviation of the mean values represent the standard error of the mean, statistical significance, $P<0.05$.

\section{Results}

High cholesterol diet and the influence of insulin treatment on the development of lipid perturbations and atherosclerosis

The control rabbits, which received cholesterol only, showed marked hyperlipaemia, especially of cholesterol esters. After two weeks of atherogenic diet, the mean plasma total fatty acids concentrations of cholesterol-fed New Zealand White rabbits were markedly increased. As shown in Fig. 1, insulin administration significantly lowered the lipaemia. Plasma total lipids, free fatty acids, triglycerides, esterified cholesterol were also lowered. By contrast, at 6,8 and 12 weeks, insulin therapy strongly

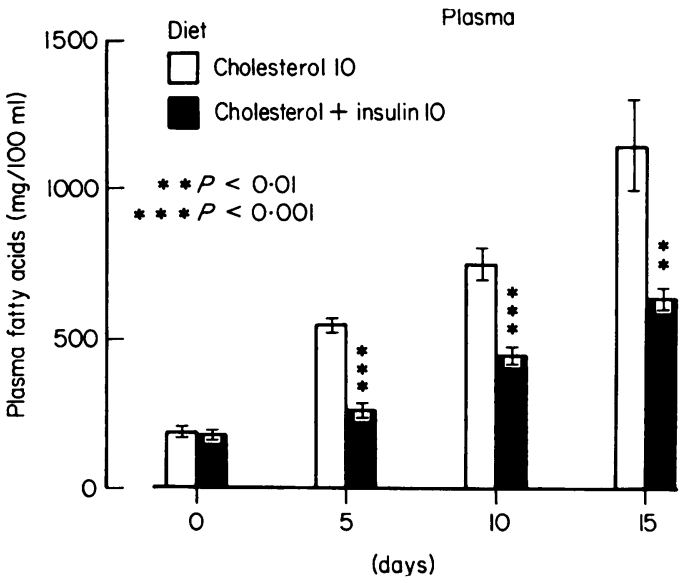

Fig. 1. The effect of insulin on plasma lipid in cholesterol-fed rabbits. Each rectangle represents the mean value and the standard error of the mean.

aggravated the plasma lipid perturbations induced by the atherogenic diet in Fawn Bourgogne rabbits (Fig. 2). Concentrations of free fatty acids, cholesterol esters and triglycerides were also higher in insulin-treated rabbits. It is worth noting that New Zealand White and Fawn Bourgogne rabbits differ markedly in their susceptibility to induction of both hypercholesterolaemia and atheroma by cholesterol diets (Marquié and Agid, 1972). New Zealand White rabbits fed a high-cholesterol diet were markedly hyperlipaemic compared with Fawn Bourgogne rabbits receiving the same dose of cholesterol. Fawn Bourgogne rabbits were used in most of the experiments, except for the one which lasted only fifteen days.

An increase in the mass of the liver was observed in the cholesterol control rabbits; the lipid accumulation was considerable at the end of the experiment. The contents of free and esterified cholesterol were particularly high, with those of phospholipids and glycerides increasing to a lesser degree. The data presented in Fig. 3 show that insulin therapy aggravated the lipid surplus regardless of the duration of treatment. All lipid fractions were raised.

Under macroscopic examination, the control animals to which only cholesterol was administered during 60 days presented lipid areas, in particular several at the level of the arch of the aorta. Histopathological examination showed degenerative changes in the superficial elastic fibres with conjunctival hyperplastic reactions; the sub-endothelial space consisted of cells rich in lipids, the 'foam cells' or 'xanthoma cells'; occasionally the elastic internal limiting membrane was fragmented; the lipophages were still very small in number in the internal zone of the tunica media. At the biochemical level, the 


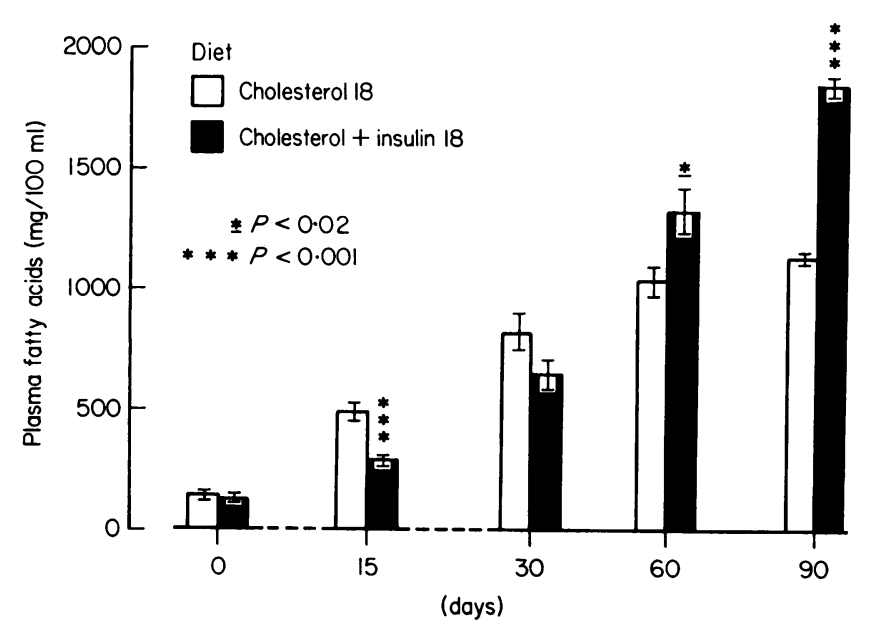

FIG. 2. The effect of long-term insulin treatment on plasma lipid in cholesterol-fed rabbits.

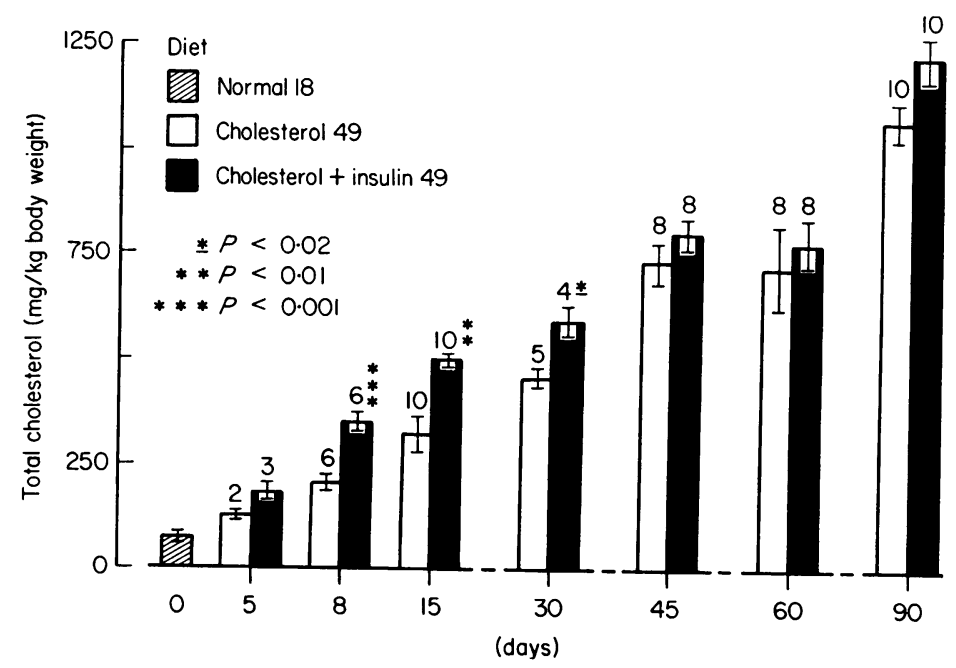

Fig. 3. Effect of insulin treatment on total cholesterol in liver of cholesterol-fed rabbits. The results are significant on the eighth, fifteenth and thirtieth day of treatment.

contents of cholesterol were $790 \pm 70 \mathrm{mg} / 100 \mathrm{~g}$ of fresh tissue as compared with $150 \pm 5 \mathrm{mg} / 100 \mathrm{~g}$ in the normal animals. Examinations of the coronary arteries showed evidence of local sub-endothelial accumulation of foam cells; the underlying tunica media not being affected.

There were generalized macroscopic lesions over the entire arterial trunk of each insulin-treated animal and this was characterized by extensive lipid areas, frequently converging at the level of the arch of the aorta. These patterns were largely confirmed at the histopathological level (index of atherosclero승 sis of $3.6 \pm 0.3$ as compared to $2.3 \pm 0.2$ in choles terol controls at the level of the thoracic aorta). In most cases, the endothelial cells were little affected $\omega$ resting on a sub-endothelial space consisting of cell packed with lipids; the elastic internal limiting membrane was fragmented; the internal zone of thee tunica media consisted of smooth muscle cells in vaded by lipids, scattered elastic elements and dis $-\frac{0}{6}$ ordered collagen fibres. The external zone of the tunica media and the adventitious tunica presented 
a normal appearance. The coronary arteries of insulin-treated rabbits showed evidence of subendothelial accumulation of foam cells; the underlying tunica media was frequently affected. The lesions were particularly clear around the small coronary vessels; the minor ramifications were completely occluded by the hyperplasia of the tunica intima. Biochemical analysis also revealed that the aortic lipid contents were significantly increased by insulin. The data in Fig. 4 show the effect in insulintreated compared with that in cholesterol-treated animals. Aortic cholesterol concentrations were particularly raised after 90 days of insulin (the mean content was $1650 \pm 65 \mathrm{mg} / 100 \mathrm{~g}$ of fresh tissue compared to $1100 \pm 32$ for controls).

\section{Influence of insulin treatment in the regression of atherosclerosis}

Control animals fed only the cholesterol diet for 60 days were then fed plain mash and sacrificed at intervals for examination of plasma, liver and arteries. The period of retrogression was that between the cessation of cholesterol feeding and the death of the animal. Hyperlipaemia decreased rapidly and returned to normal levels after 60 days. All lipid fractions (esterified cholesterol, free cholesterol, triglycerides, free fatty acids, phospholipids) also decreased. The previously existing lipid accumulation in the liver lasted for 15 days following termination of the atherogenic diet; from the fifteenth day, the lipid content gradually decreased; on the 60th day, cholesterol values were near-normal.

Insulin treatment accelerated the rate of reduction of hypercholesterolaemia; data were particularly significant on the eighth and fifteenth day (Fig. 5). Nevertheless, insulin prevented the spontaneous clearance of hepatic lipid deposits compared with controls. Insulin-treated rabbits showed a large accumulation of cholesterol in the liver for approximately 60 days.

The cholesterol content of the aortic wall increased in control animals during the first three months of normal diet (from $860 \pm 55$ to $1200 \pm 90 \mathrm{mg} / 100 \mathrm{~g}$ of fresh tissue). At the fourth month, the lipid deposits from the aorta and coronary arteries disappeared; this was accompanied by a gradual increase in the number of fibroblastic cells in the atherosclerotic lesions.

Insulin was found to alter the rate or degree of disappearance of lipid deposits in the aorta. Indeed, therapy with insulin for four months resulted in a great accumulation of lipid in the aortic wall compared with the control group (Fig. 5). Microscopic examination of the entire length of the aorta also revealed appreciable differences between the atherosclerotic lesions of insulin-treated rabbits and their controls. The atherosclerosis of the aorta produced in the insulin-treated rabbits was much more severe than in the controls; and rabbits given insulin exhibited no regression of coronary lesions. The lesions remained particularly clear around the small coronary vessels, while in more than $50 \%$ of the control rabbits the coronary arteries had a normal appearance.

\section{Discussion}

The data presented in this paper show that insulin strongly reduced plasma lipid disturbances induced by cholesterol during a short period. But the prolongation of insulin treatment aggravated hypercholesterolaemia and atherogenesis in rabbits fed a diet rich in cholesterol. The findings of the regression

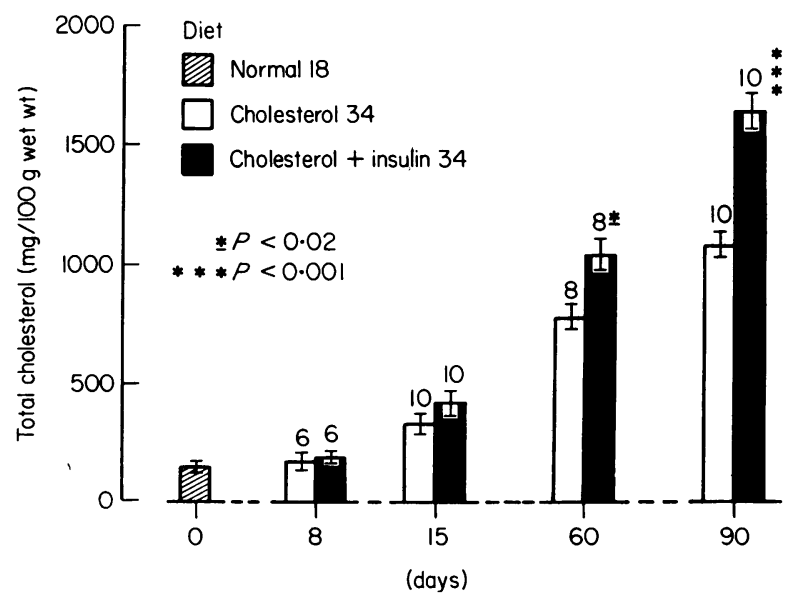

FIG. 4. Effect of insulin treatment on total cholesterol in aortic tissue of cholesterol-fed rabbits. 
Insulin after cessation of the cholesterol diet
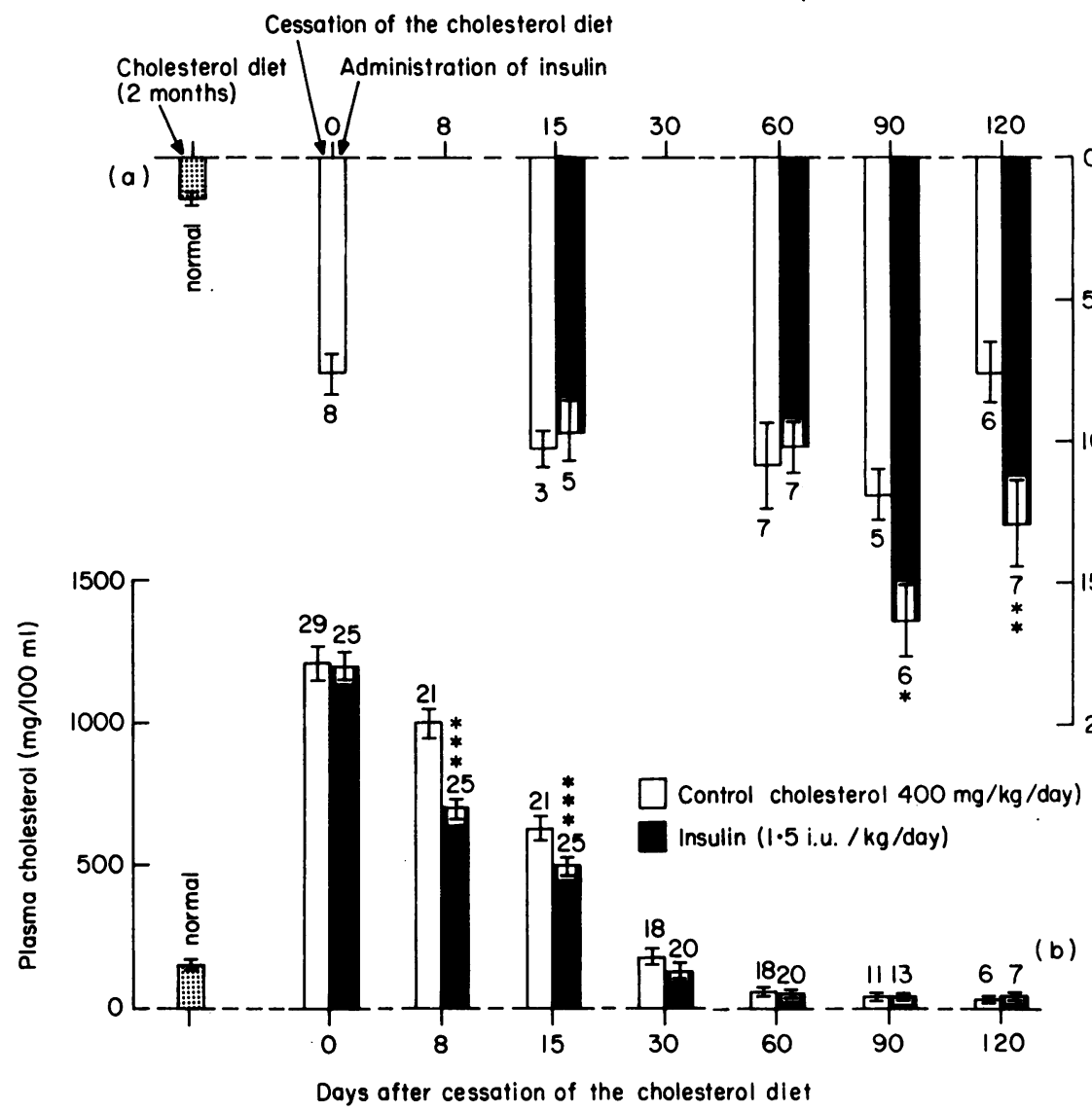

FIG. 5. The effect of insulin treatment on (a) aortic and (b) plasma cholesterol in rabbits after cessation of the cholesterol rich diet and the influence of insulin treatment.

data demonstrate that insulin, although it accelerated the rate of reduction of the hypercholesterolaemia, nevertheless aggravated the lipid infiltration of the artery walls and especially inhibited the regression of coronary atherosclerosis that usually occurs in animals transferred from a high-cholesterol diet to unsupplemented plain mash.

Findings indicating an atherogenic property of insulin have been previously reported in diabetic rabbits. In alloxan diabetic rabbits fed cholesterol, aorta atherosclerosis was much less severe than in intact animals on the same diet (McGill and Holman, 1949; Duff and McMillan, 1949; Duff and Payne, 1950; Cook, Mills and Green, 1954). However, when the diabetic rabbits were treated with insulin, severe aorta atherosclerosis occurred (Duff, Brechim and Finkelstein, 1954). There is a major difference between the previous experiments and the present one. In Duff's experiments, the rabbits were dias betic, the present ones were not. Neverthelessfindings indicate in both cases that insulin aggra@ vated aortic and coronary atherosclerosis. However, the author has shown that the insulin was associate with complete inhibition of regression of coronary lesions. It is worth noting that the control group anc. the insulin group at the weak doses used did notw differ significantly in feed intake, weight and blood glucose during the experiment. Besides, the dat from the experiment of Wilson, Martin and Hartrof (1969) demonstrate the failure of insulin therapy t $\bar{Q}$ prevent cardiovascular lesions in diabetic rats fed aq atherogenic diet. Insulin administration in cockerel\$ abolished the preventive effect of oestrogens on ${ }^{2}$ experimental atherosclerosis (Stamler, Pick andए Katz, 1960). Stout (1970) also showed the develop? ment of vascular lesions in insulin-treated chicken\$ 
fed a normal diet. The cited observations on these effects of insulin in diabetic rabbits and rats, in intact cockerels, coupled with the results of the present experiment, show that this hormone intensifies atherogenesis.

The atherogenic effect of insulin could be involved in the stimulation of lipid synthesis elsewhere in the body (Reynold et al., 1965), as lipid is a major component of the atherosclerotic plaque. Mahler (1966) and Stout $(1968,1969)$ described the acute effect of insulin on the lipid metabolism of the arterial wall in the rat. Their results are in accord with those of the present experiment in insulin-treated rabbits which had large lipid deposits in the aorta. It is evident that the process of lipid accumulation is the result of two sets of factors: one, the balance of which hinders or promotes the deposit of lipids in the arterial wall; the other, the balance of which hinders or promotes their removal after they are deposited. From the present experiments it is clear that insulin acts on the two distinct sets of factors promoting the aortic lipid deposits and hindering their removal. The action mechanism of this fundamental biological process is currently the subject of research by the author and his colleagues.

\section{References}

AlbrinK, M.J. (1959) The microtitration of total fatty acids of serum with notes on the estimation of triglycerides. Journal of Lipid Research, 1, 53.

ANitschkow, N. (1933) Experimental arteriosclerosis in animals. In: Arteriosclerosis: A Survey of the Problem (Ed. by E. V. Cowdry), chapter 10. Macmillan Co., New York.

Chen, P.S., Toribara, T.Y. \& Warner, H. (1956) Microdetermination of phosphorus. Analytical Chemistry, 28, 1756.

Christiansen, I., Deckert, T., Kuerulf, K., MitgaArd, K. \& WORNING, H. (1968) Glucose tolerance, plasma lipids and serum insulin in patients with ischaemic heart disease. Acta medica scandinavica, 184, 283.

CooK, D.L., Mills, L.M. \& Green, D.M. (1954) The mechanism of alloxan protection in experimental atherosclerosis. Journal of Experimental Medicine, 99, 119.

Duff, G.L. \& McMillan, G.C. (1949) The effect of alloxan diabetes on experimental cholesterol atherosclerosis in the rabbit. I. The inhibition of experimental cholesterol atherosclerosis in alloxan diabetes. II. The effect of alloxan diabetes on the retrogression of experimental cholesterol atherosclerosis. Journal of Experimental Medicine, 89, 611.

DufF, G.L. \& PAYNE, T.R.B. (1950) The effect of alloxan diabetes on experimental cholesterol atherosclerosis in the rabbit. III. The mechanism of the inhibition of experimental cholesterol atherosclerosis in alloxan-diabetic rabbits. Journal of Experimental Medicine, 92, 299.

DufF, G.L., BRechim, D.J.H. \& Finkelstein, W.E. (1954) The effect of alloxan diabetes on experimental cholesterol atherosclerosis in the rabbit. IV. The effect of insulin therapy on the inhibition of atherosclerosis in the alloxandiabetic rabbit. Journal of Experimental Medicine, 300, 371.

Folch, J., Lees, M. \& Stanley, G.H.S. (1957) A simple method for the isolation and purification of total lipids from animal tissues. Journal of Biological Chemistry, 226, 497.

Laurell, S. \& Tibbling, G. (1966) Colorimetric microdetermination of free fatty acids in plasma. Clinica chimica acta, 16, 57.

Leetma, H.E., Gertler, M.M., Welsh, J.J., Saluste, E. \& WHITER, H. (1968) Insulin response to glucose stress in covert ischaemic heart disease. Circulation, 38 (Suppl. 6), 124.

MAHLER, R.F. (1966) Insulin action on arterial tissue in relation to diabetes and atheroma. In: Diabetes Mellitus (Ed. by L. J. P. Duncan). University Press, Edinburgh.

MARQuí, G. \& AGID, R. (1972) Influence de la race sur la sensibilité du lapin à l'athérome expérimental. Experientia, 28, 1068.

McGill, H.C. \& Holman, R.L. (1949) The influence of alloxan diabetes on cholesterol atheromatosis in the rabbit. Proceedings of the Society for Experimental Biology and Medicine, 72, 72.

MilleR, B.F. \& Kothati, V.H. (1969) Increased activity of lysosomal enzymes in human atherosclerotic aortas. Experimental and Molecular Pathology, 10, 288.

Peters, N. \& Hales, C.N. (1965) Plasma insulin concentrations after myocardial infarction. Lancet, $\mathbf{i}, 1144$.

Renold, A.E., Crofford, O.B., Staufacher, W. \& JeanRENAUD, B. (1965) Hormonal control of adipose tissue metabolism, with special reference to the effects of insulin. Diabetologia, 1, 4.

SoMOGYI, M. (1952) Notes on sugar determination. Journal of Biological Chemistry, 195, 19.

SPERRY, W.M. \& BRAND, F. (1955) Determination of total lipids in blood serum. Journal of Biological Chemistry, 213, 69.

Stadtman, T.C. (1957) Determination of cholesterol and ergosterol by Lieberman-Burchard reaction. In: Methods in Enzymology (Ed. S. P. Colowick \& N. O. Kaplan), p. 362. Academic Press, New York.

Stamler, J., Pick, R. \& Katz, L.N. (1960) Effect of insulin in the induction and regression of atherosclerosis in the chick. Circulation Research, 8, 572.

STouT, R.W. (1968) Insulin-stimulated lipogenesis in arterial tissue in relation to diabetes and atheroma. Lancet, ii, 702.

Stout, R.W. (1969) Insulin stimulation of cholesterol synthesis by arterial tissue. Lancet, ii, 467.

Stout, R.W. \& Wallance-OWen, J. (1969) Insulin and atheroma. Lancet, i, 1078.

Stout, R.W. (1970) Development of vascular lesions in insulin-treated animals fed a normal diet. British Medical Journal, 3, 685 .

STOUT, R.W. (1971) The effect of insulin on the incorporation of sodium $(1-14 \mathrm{C})$-acetate into the lipids of the rat aorta. Diabetologia, 7, 367.

Tzagournis, M., Chiles, R., Ryan, J.M. \& Skillman, T.G. (1968) Interrelationships of hyperinsulinism and hypertriglyceridaemia in young patients with coronary heart disease. Circulation, 38, 1156.

Wilson, R.B., Martin, J.M. \& Hartroft, W.S. (1969) Failure of insulin therapy to prevent cardiovascular lesions in diabetic rats fed an atherogenic diet. Diabetes, 18, 225. 